\title{
New application of genetic algorithm in optimization of structural weights
}

\author{
Mokhtar Jalilian ${ }^{*}$, Nasser Taghizadieh ${ }^{2}$ \\ ${ }^{I}$ Master Student Civil Structures, Department of Civil Engineering, University of Tabriz, Tabriz, Iran \\ ${ }^{2}$ Assistant Professor of Faculty Departments of Civil Engineering, University of Tabriz, Tabriz, Iran
}

\begin{abstract}
It has been widely recognized that the performance of a multi-agent system (MAS) is highly affected by its organization. A large scale MAS may have billions of possible ways of organization, depending on the number of agents, the roles, and the relationships among these agents. These characteristics make it impractical to find an optimal choice of organization using exhaustive search methods. In this report, we propose a genetic algorithm aided optimization scheme for designing hierarchical structures of multi-agent systems. We introduce a novel algorithm, called the hierarchical genetic algorithm, in which hierarchical crossover with a repair strategy and mutation of small perturbation are used. The phenotypic hierarchical structure space is translated to the genome-like array representation space, which makes the algorithm genetic-operator-literate. A case study with 10 scenarios of a hierarchical information retrieval model is provided. Our experiments have shown that competitive baseline structures which lead to the optimal organization in terms of utility can be found by the proposed algorithm during the evolutionary search. Compared with the traditional genetic operators, the newly introduced operators produced better organizations of higher utility more consistently in a variety of test cases. The proposed algorithm extends the search processes of the state-of-the-art multi-agent organization design methodologies, and is more computationally efficient in a large search space.
\end{abstract}

Keywords: genetic algorithm, hierarchical crossover, information retrieval, multi-agent systems, organization design, optimization, representation, tree structures.

\section{Introduction}

The research on the organization of a multi-agent system (MAS) has attracted much interest in recent years. An organization provides a framework for activities and interactions in a MAS through the definition of agent roles, groups, tasks, behavioral expectations and authority relationships such that all the agents in the MAS can cooperate systematically and contribute to the common good of the overall system. More specifically, the organization defines which resources an agent is able to acquire, what roles/functions it takes, with which other agents it is allowed to exchange information, etc.

A proper organization for a MAS can ensure the behavior of the agents to be externally observable and make up for the major drawback of the traditional agent centered MAS in which the patterns and the outcomes of the interactions are inherently unpredictable because of the high likelihood of emergent (and unwanted) behavior [4]. Particularly, in large scale systems, to form and evolve an organization makes it possible for the system to exploit collective efficiencies and to manage emerging situations [12]. So far, a number of organization designs have been proposed for multi-agent systems [9]. Experiments and simulations have shown that various organizations employed by a system with the same set of agents may have different impacts on its performance [8][15][5][10][17][21].

Among all kinds of organizations, the hierarchical structure is one of the most common structures observed in multi-agent systems. Like human organizations, primate societies, and insect colonies, many multiagent systems can be abstracted as hierarchical, tree-like structures or sets of parallel hierarchical structures, where agents are categorized in different levels in the hierarchies [11]. Often, the level of an agent indicates its capabilities and roles. In other words, a specific level in the system consists of equally capable agents, performing similar roles. Agents at the bottom level may execute the routine tasks under the orders given by their higher-level authorities, whereas agents at the top level may assign the task, collect and assemble the returned information from their subordinates, as seen in the distributed information retrieval (IR) system described in [10].

For a large hierarchical MAS, there exist a great variety of possible ways to organize the system, which induces different agent behaviors and system characteristics. Due to the difference in the depth and the width of the hierarchy, the number of organization instances increases exponentially with the number of agents, which poses a great challenge for us to construct the most suitable organization for a given system. Although many methodologies for organization modeling have been proposed, few of them present an effective way to search for an optimal organization instance. 
In order to solve the problem, this report proposes a genetic algorithm (GA) approach as an alternative to the conventional enumeration methods for optimizing hierarchical multi-agent systems. Inspired by biological evolution processes such as selection, reproduction, and mutation, GAs are known to be robust global search algorithms for optimization and machine learning [7][2][3]. The heuristic nature of GA helps it to locate the global optimum in a vast search space. We design novel crossover and mutation operators to make the algorithm suitable for organization evolution and thereby ensure competitive performance. We tested the algorithm in an example of the IR model [10] which exhibits numerous possible organizational variants and verify its capability through simulations in different scenarios.

The rest of the report is structured as follows. Section 2 discusses the related work. In Section 3, we introduce the representation of organization employed in our algorithm, followed by the newly proposed crossover and mutation operators in Section 4. Section 5 proceeds with description of the IR model in our case study, with implementation details and experimental setup. And in Section 6, the simulation results are presented with the number of databases varying from 12 to 30 . We analyze the results by comparing the different test cases which show the impact of environment variables on the best organizations obtained. The proposed algorithm is compared with the standard genetic algorithm (SGA) with one-point crossover and twopoint crossover in terms of its search accuracy and stability. In Section 7, we further compare our algorithm with the search process of the state-of-the-art multi-agent organization design methodologies. In the last section, we conclude the report and discuss promising future research directions in this topic.

\section{Related Work}

The design of a multi-agent system organization has been investigated by many researchers. Early methodologies such as Gaia [19] and OMNI [18] aim to assist the manual design process of agent organizations. In these models the roles that agents have to play within the MAS and the interaction protocols are identified. Instead of relying heavily on the expertise of human designers, it is desirable to automate the process of producing multi-agent organization designs. In this case, a quantitative measurement of a set of metrics is essentially needed for us to rapidly and precisely predict the performance of the MAS. With these metrics we can evaluate a number of organization instances, rank them, and select the best organization without having to introduce heavy cost by actually implementing the organization designs.

In [10], the utility value was defined as the quantitative measurement of the performance of a distributed sensor network and an information retrieval system. An organizational design modeling language (ODML) was proposed and a template was constructed for each domain. Several approaches, including the exploitation of hard constraints and equivalence classes, parallel search, and the use of abstraction, have been studied in order to reduce the complexity of searching for a valid optimal organization.

Another organization designer, KB-ORG, which also incorporates quantitative utility as a user evaluation criterion, was proposed for multi-agent systems in [17]. It uses both application-level and coordination-level organization design knowledge to explore the combinatorial search space of candidate organizations selectively. This approach significantly reduces the exploration effort required to produce effective designs as compared to modeling and evaluation-based approaches that do not incorporate designer expertise.

Nonetheless, similar to ODML, KB-ORG aims at pruning the search space. However, the design knowledge alone is inadequate for the identification of an optimal design when the possible varieties of the organization structure become large.

Evolutionary based search mechanisms have been used to help the design of MAS organizations on a few occasions. For example, in [20], a GA-based algorithm is proposed for coalition structure formation which aims at achieving the goals of high performance, scalability, and fast convergence rate simultaneously. And in [13], a heuristic search method, called evolutionary organizational search (EOS), which is based on genetic programming (GP), was introduced. A review of evolutionary methodologies, mostly involving co-evolution, for the engineering of multi-agent market mechanisms, can also be found in [16]. These techniques show a promising direction to deal with the organization search in hierarchical multi-agent systems, as exhaustive methods, such as breadth-first search and depth-first search, become inefficient and impractical in a large search space.

\section{Representation of Organizations}

Generally speaking, the organization of a hierarchical MAS consists of a number of tree structures. It can either be a single tree, where the root node is the sole leader of the organization, or a set of trees, where there are several equally important leaders that communicate with each other and share the decision-making power. The intermediate nodes in a tree have the responsibility to assign tasks to their subordinates, as well as reporting the results of the accomplished tasks back to their higher-level authorities. Information exchange is only allowed in the vertical directions between higher and lower levels, and there is no interaction of agents 
horizontally, or among different hierarchies. The leaf nodes are the bottom of the structure and they complete the most basic tasks.

Optimization in such a search space can be handled by evolutionary algorithms [3], especially genetic programming, which supports populations of model structures of varying length and complexity. It has also been shown from previous studies that some well-structured trees (e.g. binary trees), with a certain number of levels and a fixed number of subordinates per node, can be represented by arrays [14][1]. Transformations are feasible as a result of their regular structures, which thereby allow the traditional crossover and mutation operators of other evolutionary algorithms, such as genetic algorithms, to take effect.

We propose an array representation of hierarchical MAS organizations which is applicable to a much broader range of hierarchical structures than just binary trees. It converts s set of hierarchical trees into a fixedlength array with integer components, which resemble gene sequences. The representation is not limited to describe a single tree, and the number of subordinates of each node need not be a constant. Unbalanced trees, in which leaf nodes are not on the same hierarchical level, can also be depicted using this representation.

\subsection{Translating Organizations into Genomes}

We assume that the hierarchical MAS considered here have the following properties. We assume that the number of leaf node agents is fixed before the search. We also assume that the maximum possible number of levels is determined. Thus, the total number of agents in the organization is bounded. Based on these assumptions, we can make use of the partition concept to convert the organization from tree structures to arrays. Let $N$ be the total number of leaf nodes or end nodes, so that the they can be numbered as $1,2, \ldots, N$ respectively from left to right. Let $M$ be the maximum tree depth (i.e. maximum height of the structure). The reason for limiting the height is that very tall structures can be slow or irresponsive, as the long path length from root to leaf increases message latency among the agents. The organization of a hierarchical MAS can be outlined by Representation 1:

$a_{1} a_{2} a_{3} \ldots a_{N-1}$ separate.

where $a_{i}$ is an integer between 1 and $M$, denoting the level number where leaf nodes $i$ and $i+1$ start to
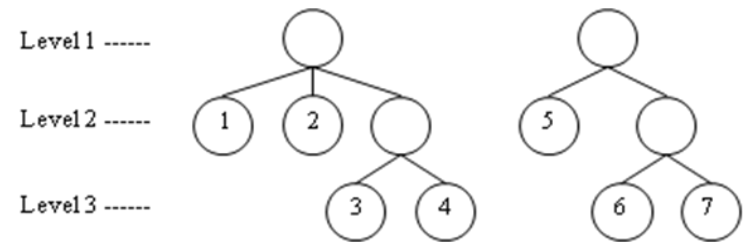

223123

Figure 1: A sample organization and its array representation. Agent nodes are displayed as circles in the figure, and leaf nodes are numbered.

An example with seven leaf nodes $(N=7)$ is illustrated in Figure 1. It consists of two trees. On Level 1, the four leaf nodes on the left and the three leaf nodes on the right separate into two trees. In other words, there is one separation between the leaf nodes 4 and 5 , so $a_{4}=1$. On Level 2, there are two leaf nodes and one intermediate node (three nodes altogether) under the left tree root, corresponding to the "2 2" (two partition numbers) to the left of the "l" in the array. The one leaf node and one intermediate node (two nodes altogether) under the right tree root give the "2" (one partition number) to the right. Both intermediate nodes on Level 2 have two leaf nodes as their subordinates (leaf nodes 3 and 4, leaf nodes 6 and 7), which are separated on Level 3, resulting in

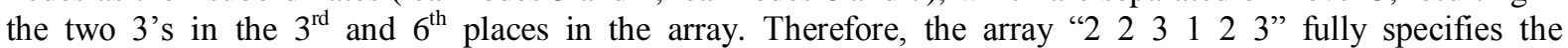
organization.

Conversely, we can also obtain an organization by interpreting the representation array. For instance, if we want to determine which level node 4 in Figure 1 sits on, we need to examine both the node's left and right neighbor. The third and forth digits in the array are " 3 " and " 1 ". It means that node 3 and node 4 are separated on Level 3. Node 4 and node 5 are separated on Level 1. As a result, we can place node 4 on Level 3 (larger number between 3 and 1). Similarly, because the fifth digit is "2", i.e. node 5 and node 6 are separated on level 2 , node 5 can be put on level 2 (larger number between 2 and 1 ). 


\section{Theorem:}

The above representation has the following properties.

(1) For every hierarchical organization instance which satisfies our assumptions in the beginning of Section 3.1, the array representation that can be generated is unique.

(2) For every representation of the above mentioned form, there is an organization instance corresponding to it.

Proof:

(1) We firstly prove the existence of an array representation for every hierarchical organization instance. The way of generating an array representation of an arbitrary hierarchical organization instance can be expressed as follows. If there are $N$ leaf nodes, we prepare $N-1$ slots. Firstly, organize the structure well so that the root nodes, intermediate nodes, and leaf nodes are on their proper levels. Secondly, we examine the separation pattern between adjacent leaf nodes one by one from left to right. Fill the slots with the level number where the adjacent leaf nodes start to separate. See Figure 1 for an example. The first two leaf nodes on the left

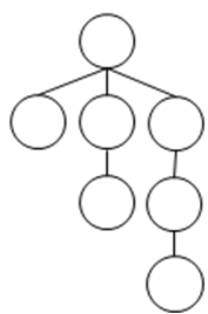

(a)

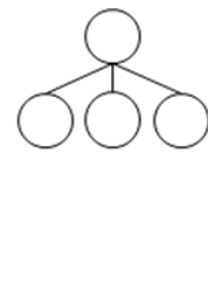

(b)

Figure 2: Organizations with the same representation.

are direct subordinates of the first tree root, i.e. on the root level (Level 1) they do not separate. However, on Level 2, they separate into different nodes. So the first number is 2 . The second slot should also be filled with number 2 because the second and third leaf nodes on the left separate on Level 2 . The third leaf node belongs to an intermediate node on Level 2 different from the second leaf node. And as the third and fourth leaf nodes are direct subordinates of an intermediate node on Level 2, they start to separate on Level 3. Number 3 should be the third number in the array representation. And so on, we can get the values, which are the level numbers, for all the slots. Together they form the required representation.

We then prove the uniqueness of the generated array representation. If array representations $a_{1} a_{2} a_{3} \ldots a_{N-1}$ and $b_{1} b_{2} b_{3} \ldots b_{N-1}$ which are derived from the same organization instance are different, there exits an $i \in\{1,2, \ldots, N\}$ such that $a_{i} \neq b_{i}$. This shows that the leaf nodes $i$ and $i+1$ separate at different levels in the two corresponding organization structures, which means the organization structures are not identical.

(2) Given an array representation with positive integers of length $L$, we would like to construct an organization instance containing $L+1$ leaf nodes as follows. Find all the digit " 1 "s in the representation (if there are any). Calculate the number of digits (greater than 1) between adjacent 1's one by one from left to right, and denote them as $n_{1}, n_{2}, n_{3}, \ldots, n_{k+1}$, where $k$ is the number of 1 's. If there are no 1 's, then $k=0$ and $n_{1}=L$. The corresponding organization has $k+1$ root nodes with $n_{1}+1, n_{2}+1, n_{3}+1, \ldots, n_{k+1}+1$ leaf nodes, respectively, from left to right. So far we have completed the root level (Level 1) of the organization. For instance, with array [2 2 $\left.\begin{array}{lll}3 & 1 & 2\end{array}\right], n_{1}=3, n_{2}=2$, i.e. there are two root nodes with 4 and 3 leaf nodes respectively. For Level 2 , we take segments with 1's and 2's as separators. These segments should only contain digits greater than 2 (if any). Like what is done for Level 1, the number of digits between adjacent separators are recorded as $r_{1}, r_{2}, r_{3}, \ldots, r_{t+1}$, where $t$ is the number of 1 's and 2's. If $r_{i}=0$, it corresponds to a leaf node; otherwise, it corresponds to an intermediate node on Level 2. After that, take segments with 1's, 2's, and 3's as separators, and repeat the steps until the greatest numbers in the representation are examined. In this way we can obtain the full organization instance.

Note that the organization instance is non-unique. Figure 2(a) illustrates an extreme case where all three leaf nodes separate on Level 2, so the representation is [2 2]. It has the same representation as the organization in Figure 2(b). When such circumstances arise, we should examine all the possible organization instances that correspond to a representation and use the best one. In the following section we explain that in the IR model, the sub-organizations having nodes with only one subordinate are uneconomical and should be simplified to achieve higher utility. Therefore, we only need to focus on the most simplified organization instance.

So far, we have established a surjective mapping from the set of all valid structure instances containing $N$ leaf nodes with maximum height $M$, denoted as $\mathbf{A}$, to the set of all arrays containing $N-1$ integer elements 
ranging from 1 to $M$, denoted as $\mathbf{B}$. Furthermore, the representation is compatible with genetic operators such as one-point, two-point or uniform crossover, i.e. the offspring generated after the crossover of individuals from set $\mathbf{B}$ still belong to set $\mathbf{B}$. Bit-wise mutation can also be applied here, so that every bit of the genome $a_{i}$ is mutated to a randomly picked different value from $\{1,2, \ldots, M\} \backslash\left\{a_{i}\right\}$ according to the user defined mutation probability.

\subsection{Simplifying Organizations}

The above representation can be applied to a general hierarchical MAS organization. For specific organization search problems, we may find it beneficial to simplify the representation in order to prune the search space and avoid unnecessary candidate evaluations of the algorithm. The simplification steps should be determined by the designer depending on the problems. Trimming, combining, and reducing of branches are easy to achieve using the proposed representation. We will give an example of how to remove redundant intermediate nodes of the IR system in Section 5.2.

\subsection{Variations of Representations}

In Section 3.1, we have assumed that the leaf nodes are homogeneous. In such circumstances, a $1 \times N-1$ array is enough to represent a hierarchical organization of a MAS. Nonetheless, in view of the circumstances where each leaf node must be treated uniquely, a second row can be added to the array representation to address the distinction resulting from permutations. This will make the representation to be in the form of a $2 \times N-1$ array (Representation 2):

$\left(\begin{array}{l}a_{1} a_{2} a_{3} \ldots a_{N-1} \\ p_{1} p_{2} p_{3} \ldots p_{N-1}\end{array}\right)$

where $\left\{a_{i}\right\}$ are still integers between 1 and $M$, denoting the level of the partition between leaf nodes $i$ and $i+1$, and $p_{1}, p_{2}, \ldots, p_{N-1}$ are a permutation of 1 to $N$ with the last number discarded. Still using the example in Figure 1 , now we use numbers $1,2, \ldots, 7$ to distinguish the mutually different leaf nodes. If in the organization they are $5,3,2,1,4,7,6$, respectively, then the representation is:

$$
\left(\begin{array}{llllll}
2 & 2 & 3 & 1 & 2 & 3 \\
5 & 3 & 2 & 1 & 4 & 7
\end{array}\right) .
$$

One may also want to design an organization in which the number of leaf node agents is not fixed beforehand. To account for varied number of leaf node agents, we may use the following Representation 3:

$$
a_{1} a_{2} a_{3} \ldots a_{N_{1}-1} \underbrace{00 \ldots 0}_{\left(N_{2}-N_{1}\right) 0 \text { 's }}
$$

where $N_{1}$ is the actual number of leaf nodes of the representation, $N_{2}$ is the maximum number of leaf nodes allowed in the organization, and the remaining positions are filled with zeros.

These variants of representations will function in the same manner as the Representation 1 when taken to go through genetic operators which are introduced next.

\section{Crossover and Mutation Operators}

The traditional one-point crossover chooses a random slicing position along the chromosomes of both parents. All data beyond that point in either solution is swapped between the two parents. The resulting chromosomes are two offspring. Though commonly used in genetic algorithms, this crossover method only influences the structure near the crossover point, as shown in Figure 3(a,b). It may not be enough to generate new offspring in large-scale systems. To speed up the evolution and increase the chance of getting a desired structure with higher utility, new crossover operators are needed. In this report, we propose a novel crossover operator - hierarchical crossover - specially designed for optimization of tree-structured organizations. 
$213342333222 \mid 12233343$

3231223122344423

One-Point Crossover

213423322344423

323122312123343
21323322123343

3231223412344423

Hierarchical Crossover

with Repair

213233221232343

323122341344423

(a) Array representation
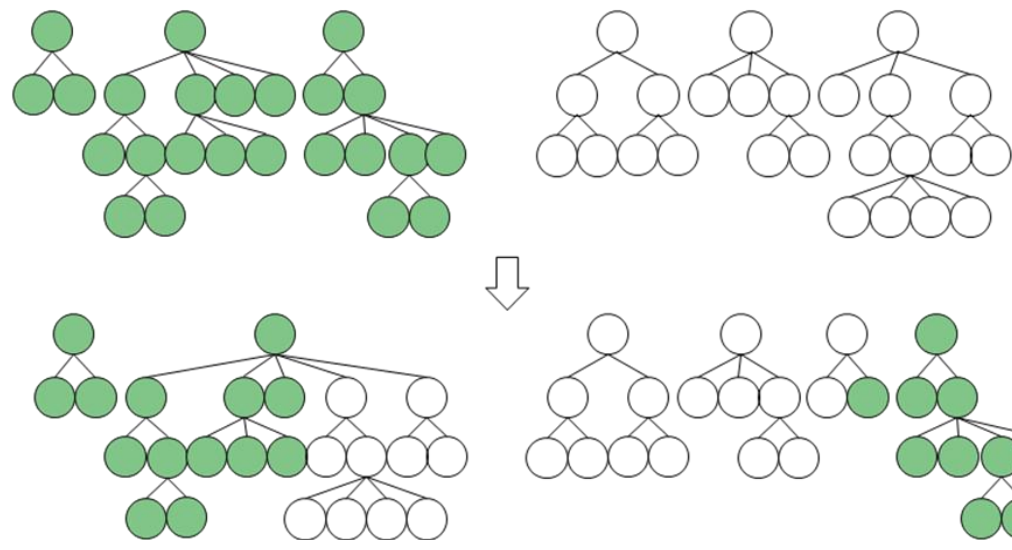

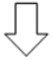

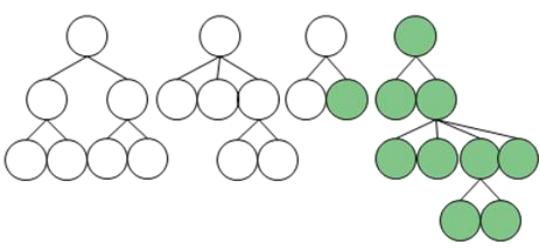

(b) One-point crossover
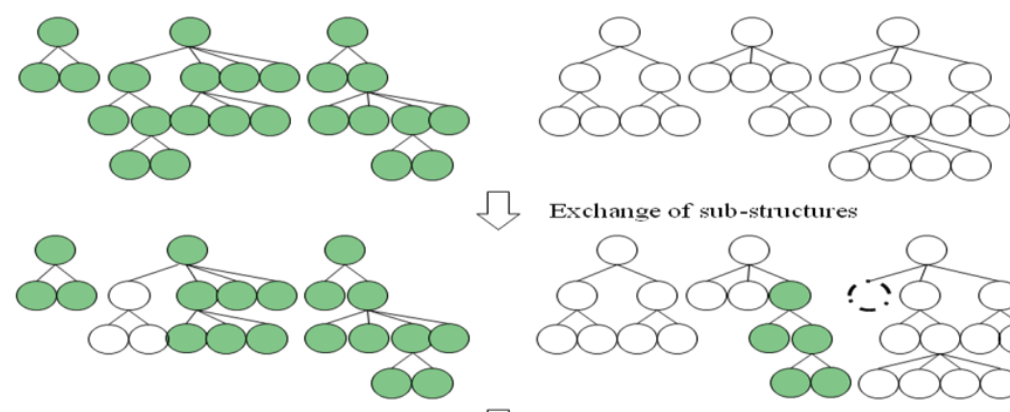

$\square$ Exchange of sub-structures
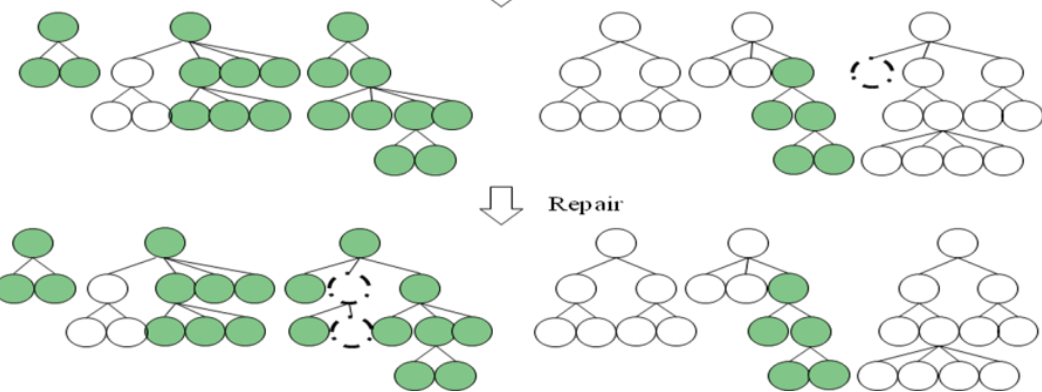

5 Repair

(c) Hierarchical crossover

Figure 3: Illustration of one-point crossover and hierarchical crossover using array representation and organization structures.

The proposed hierarchical crossover operator based on the previously described Representation 1 contains swapping of sub-organizations and a repair strategy to keep the number of total leaf nodes constant. It is implemented as follows.

First of all, we compare the number of structure levels of two randomly selected organization solutions from the population. Denote the organization with more levels as the first individual and the number of levels as $T$. Denote the organization with fewer levels as the second individual. (In the case of a tie, the order can be arbitrarily assigned.) After that, we choose a node randomly from all nodes whose level number is between 1 and $T-1$ from the first solution and denote the level number of the chosen node as $S$. Thirdly, we choose a node randomly at Level $S$, or the penultimate level, whichever is smaller, from the second solution, and exchange the sub-structures between the two solutions below the chosen nodes. If any of the solution candidates have only 
one level, we generate two random individuals of maximum tree depth instead. The exchange ensures that the two newly formed organization structures do not exceed the maximum height of their parent structures. However, the exchanged sub-structures do not necessarily contain equal number of leaf nodes. Thus, we propose the following repair strategy.

Find the solution with longer representation and randomly pick out one digit from it and insert this digit into a random slot in the other solution. Continue until the two solutions have equal length. This will guarantee the validity of the two solutions, as shown in Figure 3(a,c). Illustrated in both the array representation and the organization structures, Figure 3 displays the difference between the proposed hierarchical crossover and onepoint crossover. The pseudo code of hierarchical crossover is given in Figure 4.

To apply hierarchical crossover to Representation 2, all we need is to bundle each column and move the second row together with the first row. As for organizations in Representation 3, the repair strategy is implemented with the digits randomly picked out from non-zero locations only and until each selected organizations have the same number of leaf nodes as before.

Let parent 1 and parent 2 be the array representations of two selected parents.

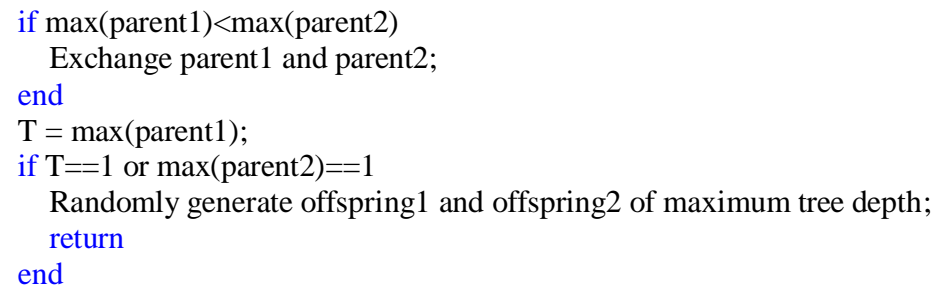

For parent1:

List all possible crossover nodes of parent1 from Level 1 till T-1;

Randomly select a node from the above list as cp1;

Record the level number of $\mathrm{cp} 1$ as $\mathrm{S}$;

Get the segments of the array representation of the sub-structure below cp1 as portion_c1;

Get the segments of the array representation to the left of the sub-structure below cp1 as portion_11;

Get the segments of the array representation to the right of the sub-structure below cp1 as portion_r1;

For parent2:

Randomly select a node cp2 from parent2 at the level number $\min (\mathrm{S}, \max (\operatorname{parent} 2)-1)$ );

Get the segments of the array representation of the sub-structure below cp2 as portion_c2;

Get the segments of the array representation to the left of the sub-structure below cp2 as portion_12;

Get the segments of the array representation to the right of the sub-structure below cp2 as portion_r2;

offspring1 = [portion_11 portion_c2 portion_r1];

offspring $2=$ [portion_12 portion_c1 portion_r2];

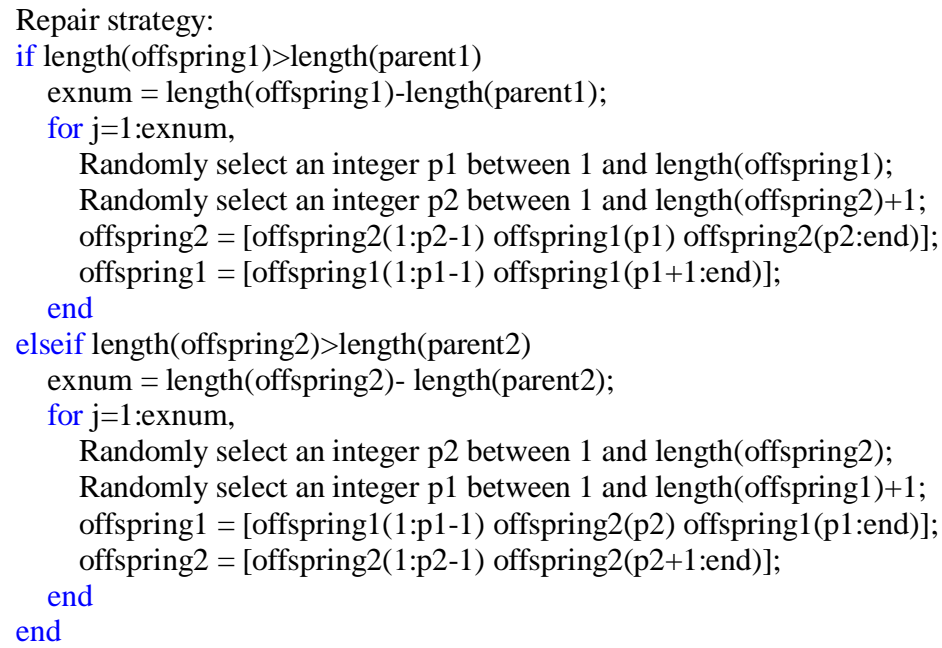

Figure 4: Pseudo code for hierarchical crossover.

As seen in Figure 3, a branch of the tree is corresponding to a piece of gene fragment. By swapping the two selected gene segments in the parents, we get two new organization instances with exchanged sub-organizations. This step is similar to two-point crossover, in which the segments between the two randomly selected crossover points of both parents are swapped to form the offspring. However, like one-point crossover, two-point 
crossover also does not concern whether the selected gene segments correspond to the whole tree branches or not. And as long as the two crossover points are determined, the segments are fixed and the locations of them in the arrays do not change. Hierarchical crossover is different from two-point crossover in that it focuses on the branches of the tree structures and only change the gene segments that refer to whole branches. Moreover, the locations of the two gene segments of the parents may differ from each other, and the repair strategy promotes population update.

In addition to the crossover method mentioned above, we use the mutation of small perturbation. It is different from bit-wise mutation in that the digit can only increase by 1 or decrease by 1 with equal probability. In the cases of the boundaries, if the perturbed digit is out of bounds, the original value is restored. The pseudo code of the mutation operator based on Representation 1 is displayed in Figure 5.

\section{The Information Retrieval Model}

In this report we will examine the algorithm in the information retrieval system [10]. A structured, hierarchical organization composed of nodes as mediators, aggregators, and databases is used to model the IR system. An agent is assigned for each node to take the corresponding functions. The information recall and the query response time are combined to form a metric to determine the utility of the organization. We will summarize the derivation of the utility function in the following section. Detailed procedures to calculate the utility can be found in [10]. In the template of the IR system shown in Figure 6, directed edges with a solid arrow represent has-a relations, and the corresponding label indicates the magnitude of that relation, and hollow-arrow edges represent is- $a$ relations.

Let offspring be the array representation of an offspring created by the crossover operator, numVar be the length of the representation, mutOps be the mutation probability, and maxTreeDepth be the maximum tree depth.

$r N=\operatorname{rand}(\operatorname{size}($ offspring, 1$)$, numVar $)<\operatorname{mutOps}$;

offspring $=$ offspring $+r N$ * $((\operatorname{rand}(\operatorname{size}($ offspring, 1$)$, numVar $)>0.5) * 2-$

$1)$;

offspring $($ offspring $==0)=1$;

offspring $($ offspring==maxTreeDepth+1) = maxTreeDepth;

Figure 5: Mutation of small perturbation.

At the top level of each hierarchy is a mediator. The user sends a query, which a randomly assigned mediator is responsible to handle. It uses the collection signatures of all the mediators to compare data sources, then routes the query to those mediators that seem appropriate. After the query has been directed through the aggregators and processed by all the databases under the selected mediators, the responsible mediator finally collects and delivers the resulting data.

\subsection{The Utility of the IR Model}

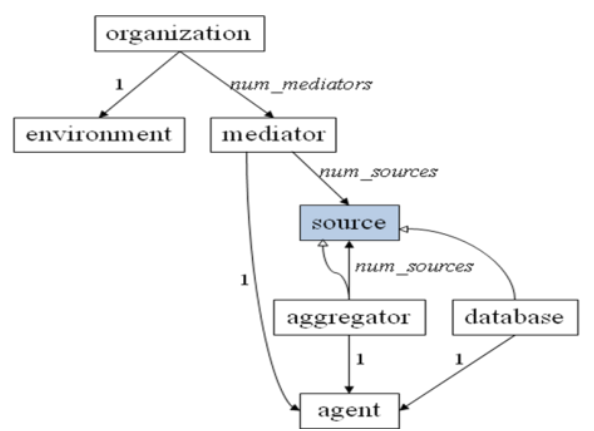

Figure 5: Organization template of the information retrieval system. [10]

According to [10], every mediator has got a rank according to its perceived response size. The one with the largest perceived response size receives rank No. 1, and the same rank is given to mediators with equal 
perceived response sizes. Mediators are chosen to be sent queries based on their ranks, resulting in the query probability $P(m)(m=1,2, \ldots$, num_mediators $)$. This is used to calculate the response recall of the organization, which is given by the following equation:

response_recall $=\frac{\sum_{m=1}^{\text {num_mediators }} P(m) \times a c t u a l_{-} r e s p o n s e \_s i z e(m)}{e n v_{\text {_topic_size }}}$

where the expectation of the system's actual response size regarding all the mediators is divided by the environmental topic size to form the value of the response recall.

The IR model assumes that queries have a Poisson arrival distribution with mean rate query rate, and each node follows the FIFO processing principle. Each database has a process service rate, defining how quickly it can process queries. Likewise, each aggregator and mediator has a response service rate, and must wait for the slowest information source before sending responds. The probability density function (pdf) and cumulative density function (cdf) of the waiting time in a database node are given as:

$$
\begin{gathered}
f_{M}(x, \lambda)=\lambda e^{-\lambda x} \\
F_{M}(x, \lambda)=1-e^{-\lambda x}
\end{gathered}
$$

where $x \geq 0$ is the waiting time and $\lambda=$ service_rate-arrival_rate. The query rate of the mediator $m$ equals query_rate $\times P(m)$, and all nodes under a particular mediator inherit the query rate of that mediator. The service rate of a database is simply its process service rate, whereas aggregators and mediators have service rate as response_service_ratelnum_sources.

The pdf and cdf of the maximum service time of a node's all sources can be generated by the following equations:

$$
\begin{aligned}
& f_{(n)}(x)=\left[\prod_{i=1}^{n} F_{i}(x)\right] \sum_{i=1}^{n} \frac{f_{i}(x)}{F_{i}(x)} \\
& F_{(n)}(x)=\prod_{i=1}^{n} F_{i}(x)
\end{aligned}
$$

where $f_{i}$ and $F_{i}$ represent the pdf and cdf of the $i^{\text {th }}$ source respectively.

The mediator and aggregator must process and aggregate the resulting data, leading to a total service time combining these two activities. The pdf and cdf of the total service time can then be determined by the convolution of the corresponding local and source distribution functions, which have the forms:

$$
\begin{aligned}
& f_{C}(x)=\sum_{i=0}^{x} f_{s}(i) \cdot f_{l}(x-i) \cdot d i s t_{-} \text {step } \\
& F_{C}(x)=\sum_{i=0}^{x} f_{s}(i) \cdot F_{l}(x-i) \cdot d i s t_{\text {_tstep }}
\end{aligned}
$$

where $x=0,1,2, \ldots$, dist_rangeldist_step, with dist_range representing the upper bound on the sampled points and dist_step the stride length between points. $f_{s}$ is the aggregate information source pdf, and $f_{l}$ and $F_{l}$ are the pdf and cdf of the waiting time for the local queuing process.

By incorporating the result propagation process and the cumulative overhead latency incurred by the message transits we can predict the expected response time of the system as a whole. And finally the utility of organization is computed by combining the aspects of response recall and response time with appropriate weights of each term as follows:

utility $=$ response_recall $\times 1000-$ response_time $/ 10$

\subsection{Simplifying the Organization Representation with Regard to the IR Model}

Since it is assumed in the IR model that all the databases in the system contain the same amount of topic data, and thus, there are no differences among the end nodes (i.e. leaves of the trees), we may directly borrow the array representation introduced in Section 3 to the IR model. Here Level 1 is the mediator level, where nodes are all mediators. The intermediate nodes correspond to aggregators, and the leaf nodes are database agents. The whole organization can be outlined by a set of trees. Exchange of information is enabled between every two root nodes and all immediate superiors and subordinates.

From a practical viewpoint, we notice that it is not necessary to include an aggregator if it only has one subordinate, because it will only increase the information transmission delay and not bring any integration advantages. Hence, if such an organization instance emerges, we can simply omit the aggregator node and reduce the organization structure by one level. 
Original representation:

315233421253143

Using " 1 " as separators:

$\underline{3} 1 \underline{523342} 1 \underline{253} 1 \underline{43}$

Using " 1 " "2" as separators:

万ु $\sqrt{3} \frac{5}{\sqrt{3}}$

$21 \underline{5} 2 \underline{334} 212 \underline{53} 1 \underline{4} 2$

ช

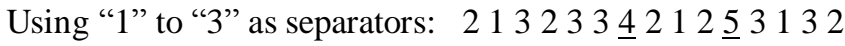

Final organization:

213233421243132
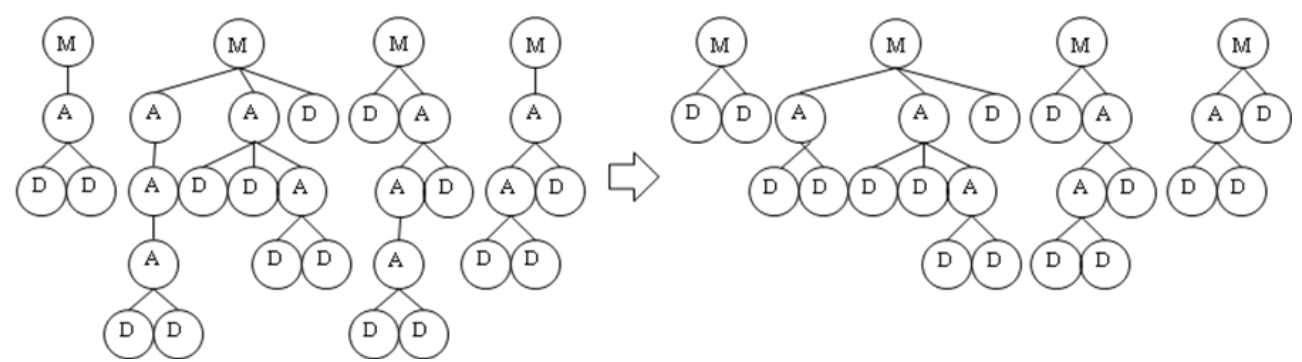

Figure 7: Simplifying the organization. Nodes $\mathrm{M}$ are mediators, nodes A are aggregators, and nodes D are databases.

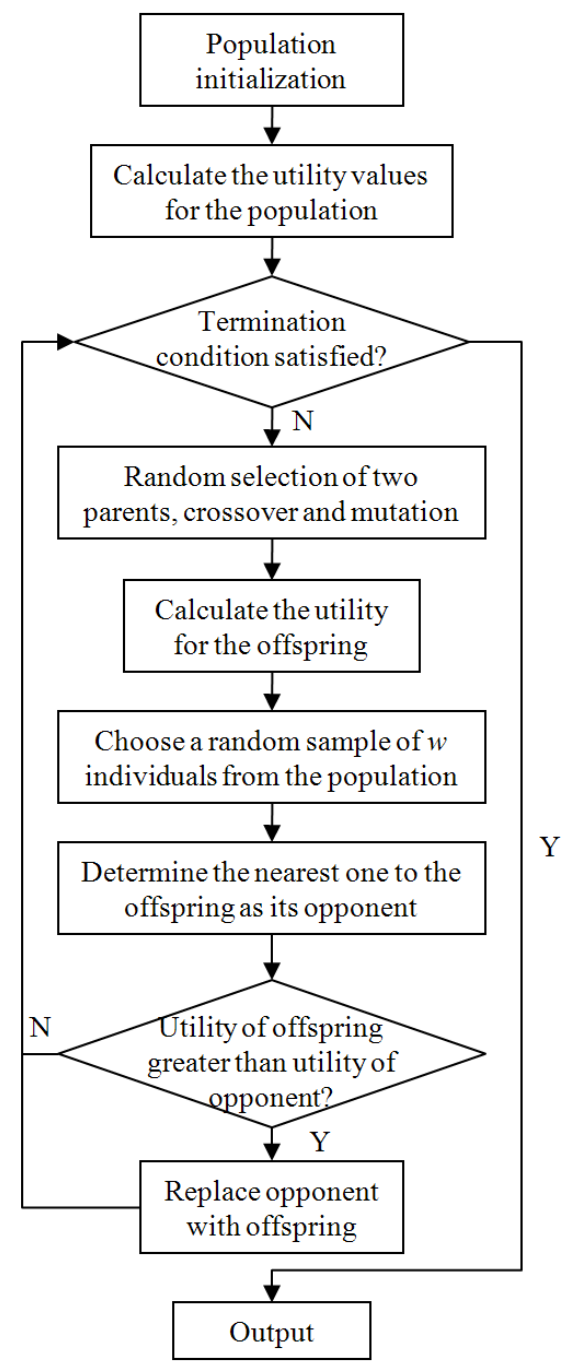

Figure 8: Flowchart of the algorithm.

Related modification can be made in the array representation, which is summarized below. Firstly, obtain all the 
segments of a genome between adjacent mediators (i.e. the integer series between 1's). Set the smallest values of these segments to 2. Secondly, obtain all the segments with 1's and 2's as separators. Set the smallest values of these segments to 3. Continue until the highest level of the organization. Figure 7 shows the detailed steps of a sample simplifying procedure. It transforms a 5-level sample organization of the IR system to a 4-level one. In the simplified organization, all mediators and aggregators have no less than two sources.

The simplifying procedure is employed to achieve higher utility. At the same time, the number of organization instances we have to evaluate for every representation is reduced to one.

\subsection{Implementation and Evaluation Criteria}

In the case study of the IR model, the optimization is carried out using genetic algorithm with population of organizations represented by arrays, the hierarchical crossover and the mutation of small perturbation as described in the above sections. The utility value serves as the fitness measure of an individual organization. If the arrival rate exceeds the service rate at one or more points, resulting in infinite queues, the fitness of the organization will be penalized. Systems with one infinite queue are considered to have fitness of 2500 , and for each additional infinite queue, another 500 is deducted from the fitness.

We recognize that there are likely multiple optimal solutions that achieve the same utility in a given system environment, owing to the symmetry of the structures. Besides, the building blocks that may lead to a good solution need to be maintained in the population. Therefore, we need a method that allows growth in several promising areas in the search space. In other words, the diversity of the population should be enhanced and over-convergence should be avoided. We increase the competition between similar individuals by applying the restricted tournament selection (RTS) method described in [6]. It helps to preserve diverse building blocks needed to locate the optimal organization. A flowchart of the algorithm is shown in Figure 8.

We compare the proposed algorithm, called hierarchical genetic algorithm (HGA), with the standard genetic algorithm using one-point crossover with bit-wise mutation (SGA1) and two-point crossover with bit-wise mutation (SGA2) in order to show the benefits of the newly introduced operators. We examine the algorithms in two aspects, the accuracy and the stability of search, which are evaluated using the parameters, average percentage relative error (APRE) and success rate (SR), respectively. They are derived using the following equations.

The percentage relative error (PRE) can be calculated by:

$\mathrm{PRE}=\left(f_{\text {best }}-f\right) / f_{\text {best }} \times 100$

where $f_{\text {best }}$ is the best known fitness value among all the runs of all the algorithms for a given test case, and $f$ is the current fitness value achieved by the algorithm. APRE is the average of the PRE values among all the independent runs of each test case.

SR is a number between 0 and 1 that denotes the ratio of the number of runs in which the best known solution is found by the algorithm to the total number of runs in each test case. Since GAs involve stochastic initialization of solution candidates, selection, crossover, and mutation, the stability of search is also an important factor that we should take account into.

We examine the test cases of $12,14,16,18,20,22,24,26,28$, and 30 databases. The maximum height of the structures is set to be 4 . The population size and the maximum number of candidate evaluations used are shown in Table 1. All algorithms use a window size $w=5$ for RTS in the population updating stage. The mutation rate is 0.1 . All the test cases involve 10 independent runs.

The environment parameters of the IR model are set as follows: message latency $=20$ milliseconds, process service rate $=10$ per second, response service rate $=20$ per second, and query rate $=3$ per second. The search set size and query set size are set to be the total number of mediators for each organization. The response recall is therefore identical (100\%) in all cases, and the utility is determined by the response time.

The best achieved fitness value in every generation is recorded and the best organization instance found after the maximum number of candidate evaluations along with its fitness are used for calculating APRE and SR. In this case study and many other applications, the computation time of the genetic operators and population updating is negligible compared to that of the candidate evaluations. Moreover, when parallel computing is used, the execution time depends on number and quality of the machines used. Therefore, we conclude that the number of candidate evaluations is more suitable as an evaluation metric than computation time. When we use the same machine, computation time is proportional to the number of candidate evaluations. All algorithms are tested in MATLAB ver. 7.9.0. 
Table 1: Configurations of HGA.

\begin{tabular}{|c|c|c|}
\hline No. DBs & Population Size & No. of Candidate Evaluations \\
\hline 12 & 50 & 2,000 \\
14 & 100 & 5,000 \\
16 & 200 & 10,000 \\
18 & 500 & 50,000 \\
20 & 500 & 50,000 \\
22 & 500 & 50,000 \\
24 & 500 & 100,000 \\
26 & 500 & 100,000 \\
28 & 500 & 100,000 \\
30 & 1,000 & 200,000 \\
\hline
\end{tabular}

\section{Experimental Results}

In this section we will firstly analyze the properties of the best solutions found by the algorithms so far. Secondly, we will demonstrate the advantage of the proposed HGA over the standard GA with one-point and two point crossover in locating the best organization of the IR system. ${ }^{1}$

\subsection{Best Organizations Found by the Algorithms}

The characteristics of the best organizations found by the algorithms are listed in Table 2, and the corresponding structures are shown in Figure 9. Since previous studies did not give comparison among the highly rated organizations in different scenarios, it should be worthwhile for us to summarize their features.

Table 2: Characteristics of the Best Organizations.

\begin{tabular}{|c|c|c|c|c|c|}
\hline No. of DBs & Representation of Best Organization & $\begin{array}{c}\text { No. of } \\
\text { Mediators }\end{array}$ & $\begin{array}{c}\text { No. of } \\
\text { Levels }\end{array}$ & $\begin{array}{c}\text { Total } \\
\text { No. of }\end{array}$ & Fitness \\
\hline 12 & 33233133233 & 2 & 3 & 18 & 860.39 \\
14 & 3233132331323 & 3 & 3 & 23 & 847.62 \\
16 & 332313323133233 & 3 & 3 & 25 & 839.20 \\
18 & 33233133233133233 & 3 & 3 & 27 & 832.27 \\
20 & 4434342443434243434 & 1 & 4 & 33 & 821.60 \\
22 & 332434341434342434434 & 2 & 4 & 37 & 813.90 \\
24 & 43434243434143434243434 & 2 & 4 & 42 & 810.13 \\
26 & 4434342443434143434243434 & 2 & 4 & 44 & 802.24 \\
28 & 443434244343414434342443434 & 2 & 4 & 46 & 795.96 \\
30 & 4434344244343441443434244343 & 2 & 4 & 48 & 790.06 \\
\hline
\end{tabular}

Firstly, we may see that there is no node with more than 6 sources in the best organization of any test case because it will cause an infinite queue in the current settings. If an aggregator has too many sources, it needs a long time to collect and analyze the information from the sources, and is thus not optimal. Secondly, most of the best found organizations are composed of the following strings: $3323,33233,443434$. These baseline structures of 5, 6, and 7 databases offer an advantage in efficiency and are assembled to constitute the best organization in a larger scale. During the evolutionary search, they are identified by the algorithms as building blocks for solutions with high fitness values. Thirdly, as the number of databases increases, the model has to deal with more distributed load. It first seeks to introduce more mediators, and later the height of the structure is increased to balance off the transmission burden of mediators. For example, 2 mediators are sufficient to handle a system with 12 databases, but for a system with 18 databases, 3 mediators are needed. And in the 20-database case, a 3level organization with 3 mediators is no longer adequate, therefore a $4^{\text {th }}$ level is added. Since the height of the structure is raised, the number of mediators is cut down to avoid unnecessary delay in assembling the data.

\footnotetext{
${ }^{1}$ As the EOS method does not contain detailed description of the algorithm, unfortunately, we are not able to compare our algorithm with EOS.
} 


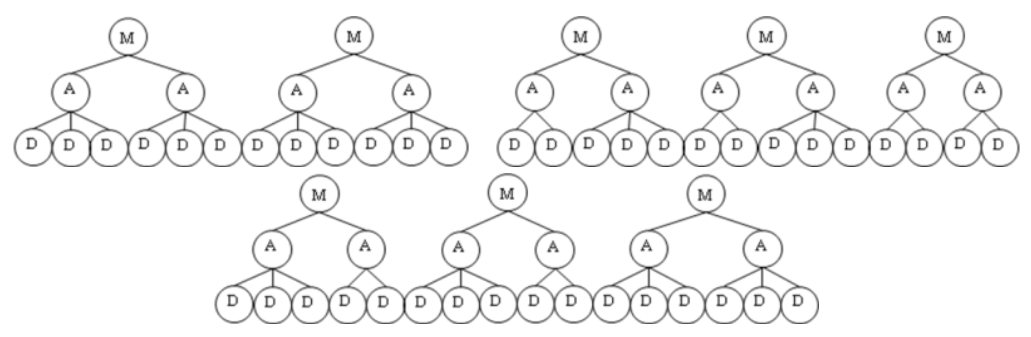

(a) 12 databases

(b) 14 databases

(c) 16 databases

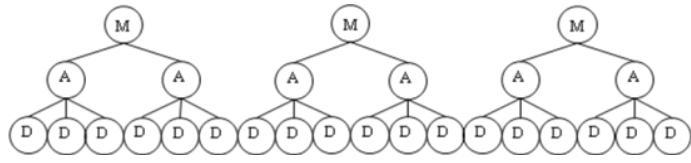

(d) 18 databases

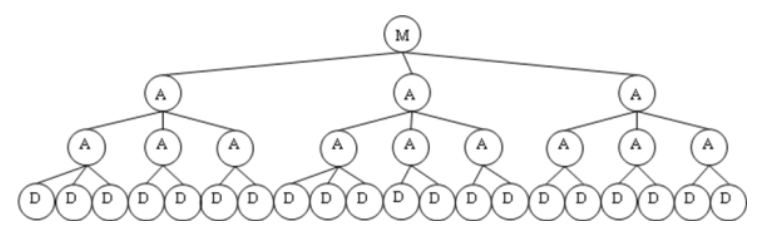

(e) 20 databases

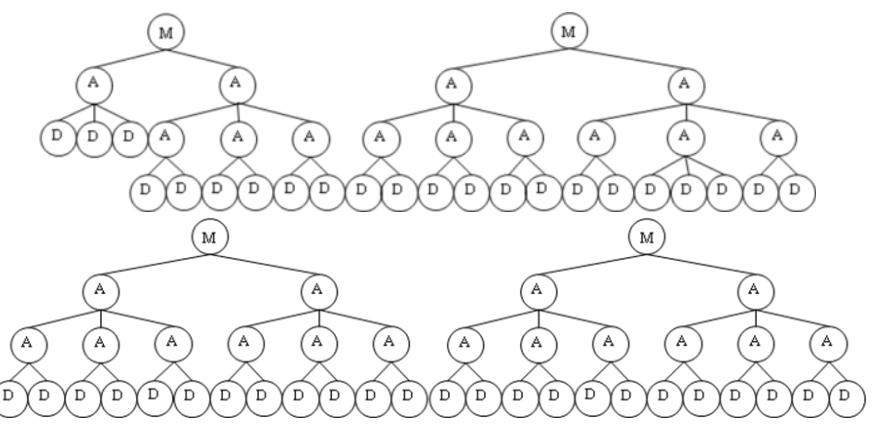

(f) 22 databases

(g) 24 databases

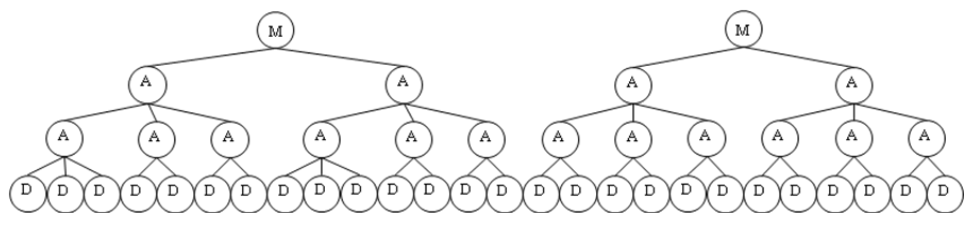

(h) 26 databases

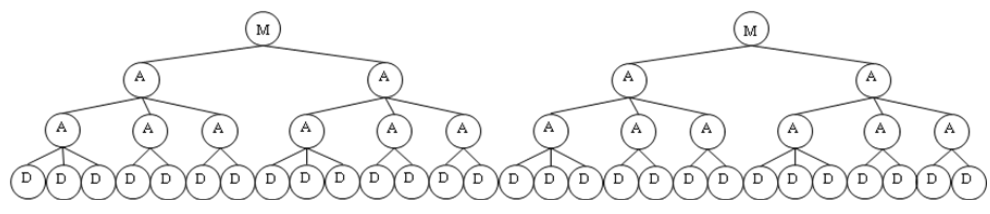

(i) 28 databases

(M)

(M)

It can be observed from Figure 9 that it is beneficial to group the databases at the bottom level as evenly as possible, which is consistent with our intuition of a good organization design. In the test cases where there are $12,18,24$, and 28 databases, balanced allocation can be realized. Perfect symmetry appears in the designs. Similar efforts are made in the test cases of 14, 16, 20, 26, and 30 databases. Note that for the latter two instances, the two mediators process different number of databases, however the second-level aggregators have exactly the same subordinate structures. The organizations shown in Figure $9(\mathrm{~h} \& \mathrm{j})$ achieve higher fitness values than the organizations with both mediators having the same number of databases, which can be represented as

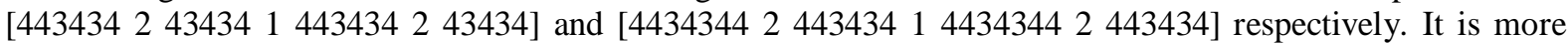
interesting to investigate the case where there are 22 databases. The tradeoff is so difficult and eventually 
unbalanced organization wins. Moreover, putting two or three databases at the penultimate level emerges as a good choice in this kind of situations.

\subsection{Comparison of Results}

Table 3 shows the APRE of SGA1, SGA2, and HGA in the 10 test cases, and the SR values are displayed in Table 4. The best value for each test case is highlighted. It can be observed that the accuracy of the proposed HGA is better than SGA1 and SGA2 in 9 out of the 10 cases. Only in the 18-database case, SGA2 outperforms SGA1 and HGA in terms of APRE.

Table 3: Average Percentage Relative Error.

\begin{tabular}{|c|c|c|c|}
\hline No. DBs & SGA1 & SGA2 & HGA \\
\hline 12 & 0.1103 & 0.1122 & $\mathbf{0 . 0 3 7 0}$ \\
14 & 0.0090 & 0.0460 & $\mathbf{0}$ \\
16 & 0.0966 & 0.0869 & $\mathbf{0}$ \\
18 & 0.0940 & $\mathbf{0 . 0 3 7 2}$ & 0.0505 \\
20 & 0.1150 & 0.3076 & $\mathbf{0 . 0 7 4 9}$ \\
22 & 0.2037 & 0.3085 & $\mathbf{0 . 0 0 3 1}$ \\
24 & 0.3376 & 0.4914 & $\mathbf{0 . 0 4 0 6}$ \\
26 & 0.1556 & 0.3494 & $\mathbf{0}$ \\
28 & 0.2104 & 0.5307 & $\mathbf{0 . 0 0 6 7}$ \\
30 & 0.2470 & 0.4825 & $\mathbf{0}$ \\
\hline
\end{tabular}

Regarding the search ability, HGA also has an advantage over SGA1 and SGA2 in the majority of the test cases. The superiority of HGA is more pronounced in larger-scale organizations which contain more than 20 database nodes. In those cases, SGA1 and SGA2 fail to locate the best known organization instances for most of the time, whereas the proposed HGA still maintains high SR values of 90\%-100\%. This proves that HGA uses fewer candidate evaluations to locate the best organization than the conventional GAs. Given that the candidate evaluations are very computationally expensive in many real-world systems, it is beneficial to use HGA in such circumstances.

Table 4: Success Rate.

\begin{tabular}{|c|c|c|c|}
\hline No. DBs & SGA1 & SGA2 & HGA \\
\hline 12 & 0.5 & 0.5 & $\mathbf{0 . 8}$ \\
14 & 0.8 & 0.7 & $\mathbf{1}$ \\
16 & 0.7 & 0.8 & $\mathbf{1}$ \\
18 & $\mathbf{0 . 8}$ & $\mathbf{0 . 8}$ & $\mathbf{0 . 8}$ \\
20 & $\mathbf{0 . 5}$ & 0.1 & 0.3 \\
22 & 0.1 & 0 & $\mathbf{0 . 9}$ \\
24 & 0.2 & 0 & $\mathbf{0 . 9}$ \\
26 & 0.4 & 0.1 & $\mathbf{1}$ \\
28 & 0.2 & 0 & $\mathbf{0 . 9}$ \\
30 & 0.2 & 0.1 & $\mathbf{1}$ \\
\hline
\end{tabular}

The non-parametric Wilcoxon signed-rank test is performed to judge whether there is a statistically significant difference between HGA and SGA1/SGA2. As a pair-wise test in a multi-problem scenario, we use all the APRE values of each algorithm as sample vectors. The null hypothesis $H_{0}$ is set as "there is no difference between HGA and SGA1/SGA2 in terms of the APRE values." Accordingly, the alternative hypothesis $H_{1}$ is "The two methods are significantly different." A significance level of 0.05 is implemented, i.e. if the p-value of the test turns out to be less than 0.05 , the algorithms involved are considered to have different performance, and the smaller the p-value is, the more distinct they are from each other. We get that the APRE values of HGA is different from those of SGA1 at the p-value of 0.001953 and is different from those of SGA2 at the p-value of 0.003906 , which suggests the proposed algorithm is statistically better than both SGAs.

The performance graphs of the median runs (i.e. the $5^{\text {th }}$ best runs in our experiment) of SGA1, SGA2, and HGA are shown in Figure 10. Owning to the specially designed genetic operators, HGA is able to locate good solutions faster in most of the circumstances. When the number of databases is larger (especially over 20 databases), HGA regularly scores higher fitness than SGA1 and SGA2 when the same number of candidate 
evaluations is used. It is also able to find better organizations within the maximum number of candidate evaluations. From Figure 10(f,g,h,i,j) we can see, HGA has a remarkable advantage over SGA1 and SGA2 in the convergence speed

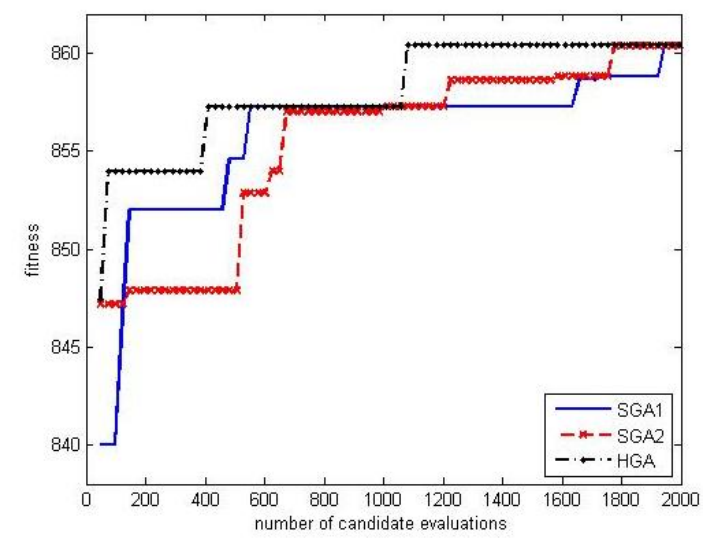

(a) 12 databases

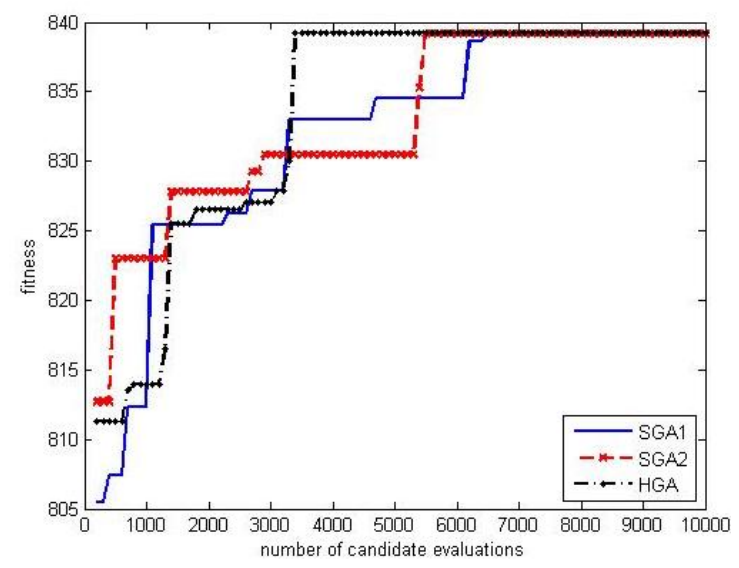

(c) 16 databases

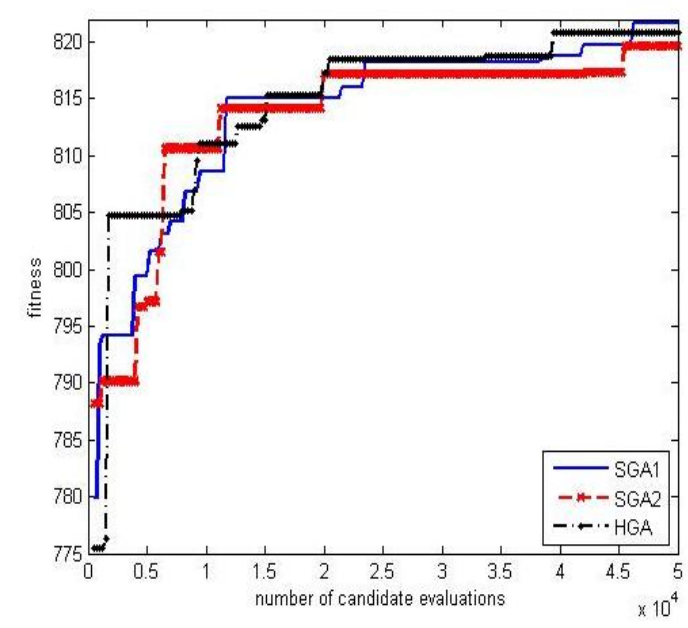

(e) 20 databases

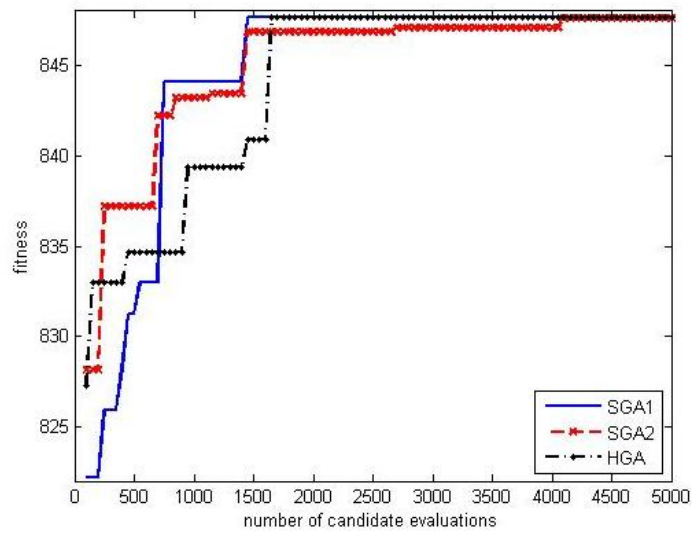

(b) 14 databases

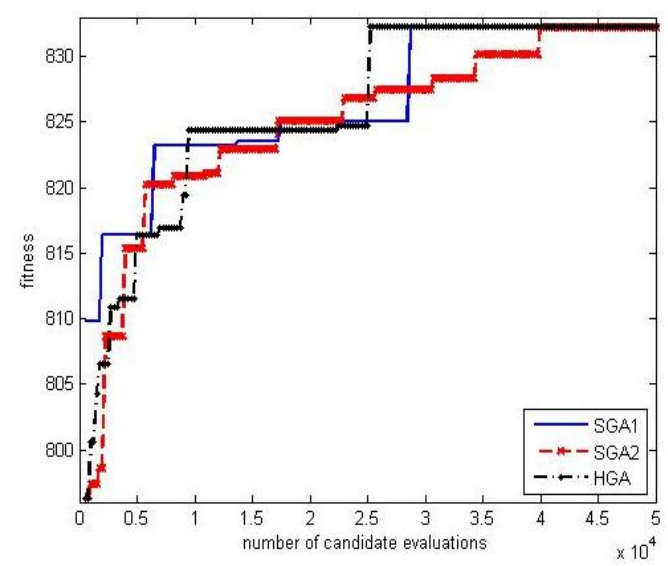

(d) 18 databases

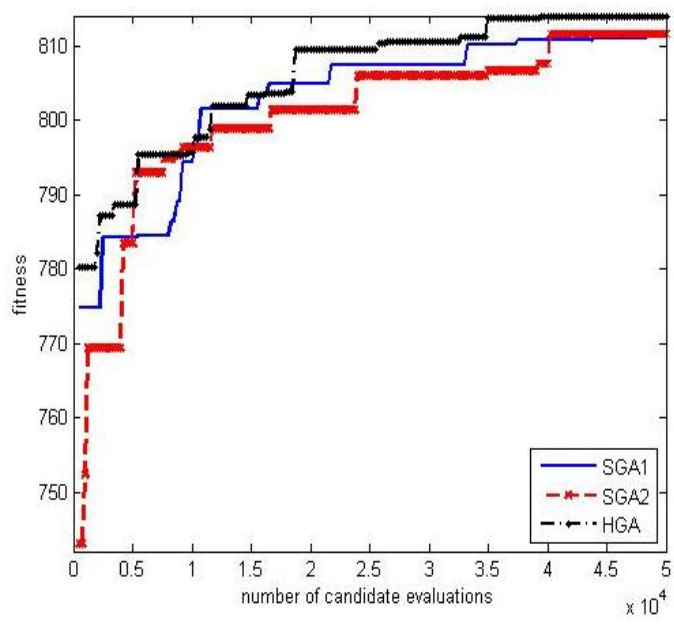

(f) 22 databases

Figure 10: Performance graph. 


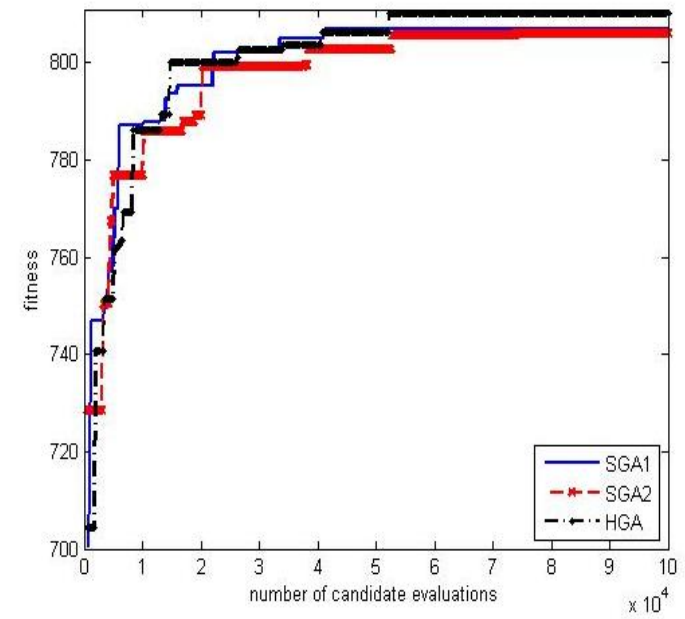

(g) 24 databases

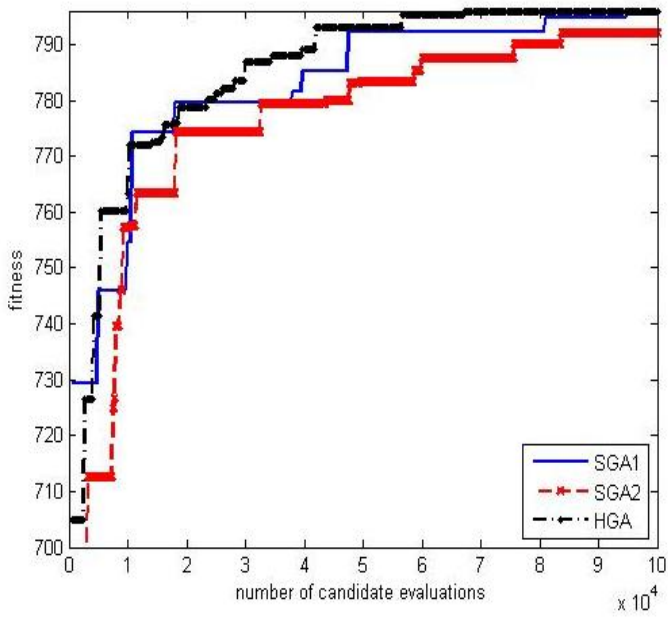

(i) 28 databases

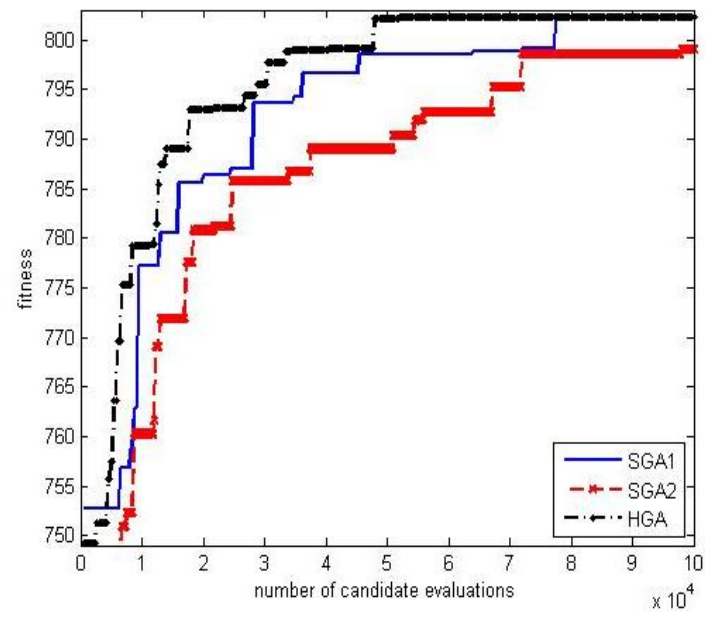

(h) 26 databases

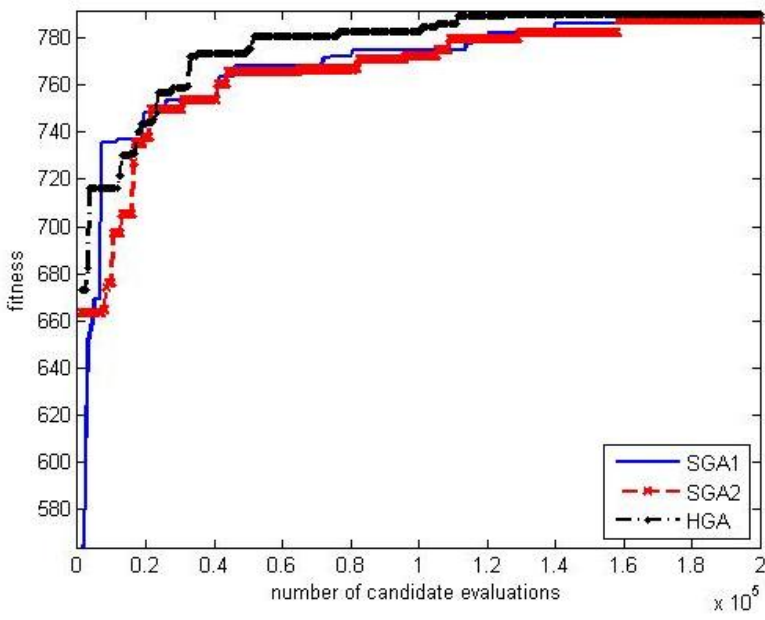

(j) 30 databases

Figure 10 (cont.): Performance graph.
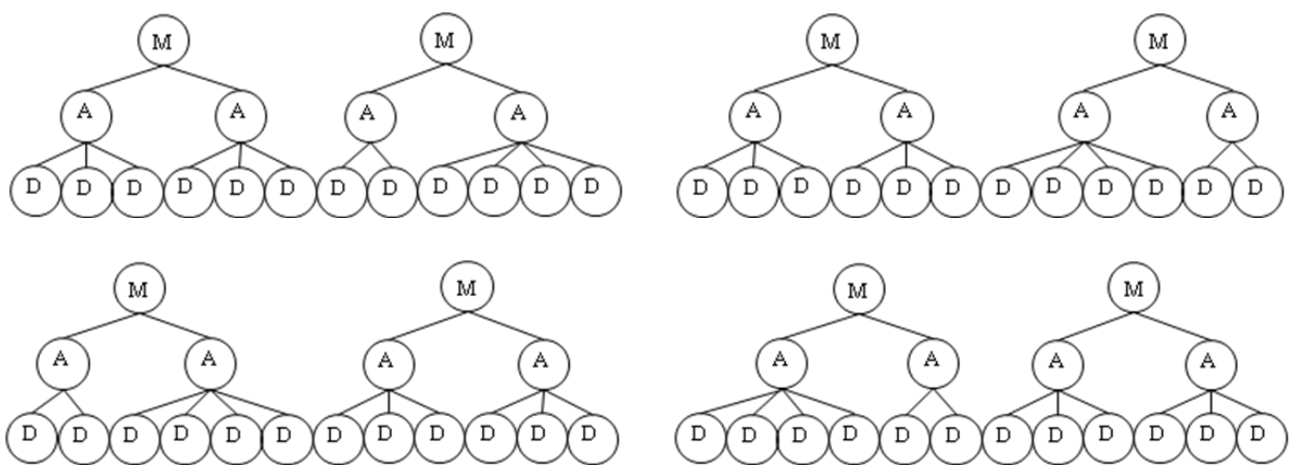

Figure 11: An Example of equivalent organizations in ODML.

\section{Comparison of HGA with the State-of-the-Art Multi-Agent Organization Design Methodologies}

While we have demonstrated the advantage of HGA's newly introduced operators over the traditional GA operators, it is interesting to investigate how HGA performs compared with the search processes of the state-of-the-art multi-agent organization design methodologies. In this section we will explore the hierarchical 
IR system using ODML [10] and KB-ORG [17] that are previously mentioned in Section 2. Results are given following the experimentation in Section 5.3.

\subsection{Comparison with ODML}

In ODML, four approaches are listed to assist the search process. They are the exploitation of hard constraints, equivalence classes, parallel search, and model abstraction. Rather than going through a decision tree to verify whether an organization instance satisfies the hard constraints of the problem as ODML does, our algorithm incorporates the array representation that already ensures the satisfaction of constraints in maximum height of the structure and the number of databases in the system. Parallel search and model abstraction are also intuitively used in HGA.

In ODML, the agents are treated in three equivalence classes: the mediators, the aggregators, and the databases. Within the same class, the characteristics of the agents do not distinguish between each other. In other words, choosing any agent in the "mediators" group for a role of mediator in the IR organization is the same. Moreover, the number of organization alternatives can be cut down by discarding organizations which are equivalent to an existing one in the candidate pool. For instance, the organizations shown in Figure 11 are equivalent in ODML in that their utility will be exactly the same, and only one should be kept as an evaluation candidate.

Based on these notions, we have calculated the number of evaluations needed for ODML in the 10 test cases as in Section 5.3, with exploited hard constraints of the number of database nodes from 12 to 30 and the maximum height of the structure equaling 4. All nodes in the organizations (expect the leaf nodes) should have a minimum of two subordinates. Details are shown in Table 5. It confirms that the number of organization instances increases exponentially as the number of leaf node agents increases, despite the truncation of redundant equivalent organizations. The total number of evaluations can be approximated as $\boldsymbol{O}\left(2.1^{N}\right)$, where $N$ is the number of leaf nodes. Comparing Table 5 with Table 1, we can see that HGA uses much fewer candidate evaluations than ODML does. Especially, when the number of databases becomes larger, the fraction of the number of candidate evaluations needed by HGA to the total number of candidate evaluations becomes smaller and smaller. This saves a great amount of computation burden, as the computation of utility functions can be extremely expensive.

Table 5: Number of organization Evaluations Needed for ODML.

\begin{tabular}{|c|c|c|c|}
\hline No. DBs & No. of Evaluations & No. DBs & No. of Evaluations \\
\hline 12 & 4,304 & 22 & $9,675,949$ \\
14 & 20,699 & 24 & $43,663,703$ \\
16 & 98,186 & 26 & $195,062,099$ \\
18 & 459,311 & 28 & $863,372,191$ \\
20 & $2,120,799$ & 30 & $3,788,734,984$ \\
\hline
\end{tabular}

It should be noted that the proposed HGA is compatible with all the above mentioned search space reducing measures, however, we maintain the equivalent organizations as in Figure 11, for they may contribute to finding an optimal solution of the test problems. This compromise results in a larger search space for HGA, whereas in ODML, the elimination of redundant equivalent organizations helps to narrow down the search range to a great extent. When the number of equivalent organizations is prevailing, ODML should have an advantage benefited from the elimination measure. Nevertheless, in the studied system, HGA still manages to evolve the population of organizations at a reasonable pace, and it spares the computation time for branch pruning at the same time.

\subsection{Comparison with KB-ORG}

KB-ORG has also placed much effort on reducing the search space. Different from ODML, it emphasizes the use of design knowledge in application and coordination of roles and design functions. With good knowledge, a system can be designed with relatively affordable cost. However, in certain cases, design knowledge is hard to acquire. It largely depends on the level of expertise of the designer. A barely trained designer may have little experience to rely on when he or she tries to construct an organization for a multi-agent system under the guideline of KB-ORG. Design knowledge is not guaranteed to be accurate. When taking a greedy approach in a certain decision step, the search process may leave out the optimal solutions. In addition, design knowledge needs to be updated following the change of environmental variables. If the environmental variables are altered, previous knowledge may not be applicable anymore; instead, new knowledge should be added to help the organization design.

In the IR model, the utility of the organizations does not involve spatial contents, and every role has only got one kind of agent to perform, so no extra knowledge is required in either spatial proximity of the agents 
or role-agent binding. The main difficulty lies in the coordination of agents, e.g. how many levels of hierarchy is needed. Assume that the designer has successfully searched out the best organizations for 12, 14, 16, and 18 databases. He may think that a 3-level hierarchy is best for the 20-databse case. This will reduce the search space to 58,327 organizations, which is only $2.75 \%$ of ODML's search space, but, it will miss out the highest rated organization, which is 4-leveled with the utility of 821.60. The best 3-level organization can be expressed with our proposed representation as [33233 13323313233233 ], with the utility of 814.11, which is worse than the worst utility (820.01) found by HGA within 50,000 evaluations in all runs. On the other hand, if the designer reaches at a relaxed bound of structure height of either 3 or 4 for the 20-database case, the number of organization evaluations will mount to 2,120,662.

Let us further assume that the designer not only has the knowledge about the vertical depth of the organization structure, but also has some knowledge about its horizontal size. If in the 22-database case, it can be speculated that the organizations with 4 levels and 2 mediators are optimal, the designer is faced with a search space of 3,384,278 options without duplicate. And for organizations with 24 databases, 4 levels, and 2 mediators, the number is $12,686,252$. If it can be speculated furthermore that the highest rated organization is made up of 4 levels, 2 mediators, with every mediator having 2 subordinate agents, the number of evaluations needed for KB-ORG will be 282,812 and 800,996 for the test cases with 22 and 24 databases respectively, whereas, for HGA, only 50,000 and 100,000 evaluations are needed to reach a $90 \%$ success rate. Although design knowledge has brought us convenience in searching for the highest rated organization in these test cases, it is far from satisfactory. In contrast, our algorithm searches for the highest rated organization in a heuristic way. It is able to handle these test cases without the assistance of external expertise.

\section{Conclusion and Future Work}

We have proposed a novel genetic algorithm based approach to solve the problem of designing the best organization in hierarchical multi-agent systems. Complementary to existing methodologies that emphasize on the pruning of the search space, our algorithm uses a bio-inspired evolutionary approach to lead the search to promising areas of the search space, and is thus suitable for optimizing multi-agent systems with a great variety of possible organizations where designer expertise alone is not enough or hard to acquire. In the example of the information retrieval system, we have empirically proved that the algorithm is able to discover competitive baseline structures in different systems and assemble them to obtain the highest rated structure from a magnitude of up to $10^{9}$ organization alternatives. In particular, we propose the use of hierarchical crossover and mutation of small perturbation to add to the advantage of our algorithm. The new crossover and mutation methods help HGA enhance the search efficiency greatly, promoting its performance both in accuracy and stability of search.

With necessary modifications, the proposed algorithm is applicable to other models as well. It can be used to optimize any tree-based hierarchical organizations of multi-agent systems, given that proper fitness values are assigned. Application areas include scenario tree and decision tree optimization. On the other hand, the proposed array representation can also be used for other forms of MAS organizations, such as holarchies. It is worthwhile to further examine the performance of the algorithm for systems with non-uniform leaf nodes and unfixed number of leaf nodes using Representation 2 and 3. In subsequent studies, we will investigate the efficiency of the proposed approach in larger-scale MASs involving a massive number of agents.

\section{References}

[1]. Aranha, C., and Iba, H. 2009. The memetic tree-based genetic algorithm and its application to portfolio optimization. Memetic Computing 1(2): 139-151.

[2]. Bäck, T. 1996. Evolutionary Algorithms in Theory and Practice: Evolution Strategies, Evolutionary Programming, Genetic Algorithms. Oxford University Press US.

[3]. De Jong, K. A. 2006. Evolutionary Computation: A Unified Approach. Cambridge, MA: MIT Press.

[4]. Ferber, J., Gutknecht, O., and Michel, F. 2003. From agents to organizations: an organizational view of multi-agent systems. In: Lecture Notes in Computer Science, 2935, Proc. Agent-Oriented Software Engineering 2003: 214-230.

[5]. Fernández, A., and Ossowski, S. 2008. Exploiting organisational information for service coordination in multiagent systems. In Proceedings of the 7th International Conference on Autonomous Agents and Multiagent Systems (AAMAS), 257-264, Estoril, Portugal.

[6]. Harik, G. R. 1995. Finding multimodal solutions using restricted tournament selection. In Proceedings of the 6th International Conference on Genetic Algorithms, 24-31. San Francisco, CA: Morgan Kaufmann Publishers Inc.

[7]. Holland, J. H. 1975. Adaptation in Natural and Artificial Systems. Ann Arbor, MI: University of Michigan Press.

[8]. Horling, B., and Lesser, V. 2005. Analyzing, modeling and predicting organizational effects in a distributed sensor network. Journal of the Brazilian Computer Society 11(1): 9-30.

[9]. Horling, B., and Lesser, V. 2005. A survey of multi-agent organizational paradigms. The Knowledge Engineering Review 19(4): 281-316.

[10]. Horling, B., and Lesser, V. 2008. Using quantitative models to search for appropriate organizational designs. Autonomous Agents and Multi-Agent Systems 16(2): 95-149.

[11]. Kirley, M. 2006. Dominance hierarchies and social diversity in multi-agent systems. Proceedings of the 8th Annual Conference on Genetic and Evolutionary Computation (GECCO), 159-166. Seattle, Washington, USA. 
[12]. Lesser, V. 1998. Reflections on the nature of multi-agent coordination and its implications for an agent architecture. Autonomous Agents and Multi-Agent Systems, 1: 89-111.

[13]. Li, B., Yu, H., Shen, Z., and Miao, C. 2009. Evolutionary organizational search. In Proceedings of the 8th International Conference on Autonomous Agents and Multiagent Systems - Volume 2, 1329-1330. Budapest, Hungary.

[14]. Nan, G., Li, M., and Kou, J. 2005. Multi-level genetic algorithm (MLGA) for the construction of clock binary tree. In Proceedings of the 2005 Conference on Genetic and Evolutionary Computation, 1441-1445. Washington DC, USA: ACM.

[15]. Okamoto, S., Scerri, P., and Sycara, K. 2008. The impact of vertical specialization on hierarchical multi-agent systems. In Proceedings of the 23rd AAAI Conference on Artificial Intelligence, 138-143.

[16]. Phelps, S., McBurney, P., and Parsons, S. 2010. Evolutionary mechanism design: a review. Autonomous Agents and Multi-Agent Systems, 21: 237-264.

[17]. Sims, M., Corkill, D., and Lesser, V. 2008. Automated organization design for multi-agent systems. Autonomous Agents and MultiAgent Systems 16(2): 151-185.

[18]. Vázquez-Salceda, J., Dignum, V., and Dignum, F. 2005. Organizing multiagent systems. Autonomous Agents and Multi-Agent Systems, 11:307-360.

[19]. Wooldridge, M., Jennings, N. R., and Kinny, D. 2000. The Gaia methodology for agent-oriented analysis and design. Autonomous Agents and Multi-Agent Systems, 3: 285-312.

[20]. Yang, J. and Luo, Z. 2007. Coalition formation mechanism in multi-agent systems based on genetic algorithms. Applied Soft Computing, 7: 561-568.

[21]. Zafar, H., Lesser, V., Corkill, D., and Ganesan, D. 2008. Using organization knowledge to improve routing performance in wireless multi-agent networks. In Proceedings of the 7th International Conference on Autonomous Agents and Multiagent Systems - Volume 2, 821-828. 